\title{
HOSPITAL DAS CLÍNICAS
}

\author{
PROF. BENEDICTO MONTENEGRO
}

Diretor da Faculdade de Medicina da Universidade de S. Paulo

Fundada a Faculdade de Medicina de S. Paulo, foi desejo de seu organizador e primeiro diretor, o inesquecivel mestre Arnaldo Vieira de Carvalho, que todas as suas dependências fossem abrigadas em prédio próprio, adequado às necessidades sempre crescentes, do ensino e condizentes com o progresso vertiginoso desta grande capital paulista.

Veiu ao seu encontro a Fundação Rockefeller, essa benemérita instituição que tanto tem contribuido para o bem estar da humanidade, em todos os recantos do globo, oferecendo-lhe uma determinada soma, sujeita essa oferta a várias condições, entre as quais a do Governo do Estado destinar igual quantia para a construção dos laboratórios que deveriam abrigar as cadeiras fundamentais.

Esta oferta foi feita em 1916 pelo Dr. Richard M. Pearce, diretor da divisão de Educação Médica da Fundação, que justificou sua escolha, depois de ter visitado muitas Faculdades de Medicina da América do Sul, porque a de S. Paulo encontrava-se ainda em formação, isenta dos erros e defeitos e das dificuldades que pesavam sobre as demais e tambem, porque aqti ele havia encontrado dois de seus antigos discipulos da Universidade de Pennsylvania, o Prof. Alexandrino Pedroso, de saudosa memória e quem estas linhas subscreve.

-Mas o espírito publico e especialmente o de noșsos governantes, não estava preparado para receber dadiva tão generosa, sem desconfiar de seus intuitos e por isso, através de vários governos estaduais, o assunto foi relegado ao olvido, até que, em 1925, na diretoria do Prof. Pedro Dias da Silva, sendo presidente do Estado o Dr. Carlos de Campos, é que foi nomeada uma comissão composta dos Profs. Luiz de Rezende Puech, Ernesto de Souza Campos e Benedito Montenegro, para visitar as principais Escolas de Medicina da Europa e dos Estados Unidos e estudar um plano completo para construção e instalação de todos as dependências desta Faculdade. Dessa viagem e desses estudos, resultou o edifício magestoso que se levanta imponente na Avenida Dr. Arnaldo, a testemunhar o acerto e o carinho com que foram resolvidos, dentro das possibilidades 
orçamentarias, todos os problemas do ensino das matérias básicas do curso médico.

Todavia, desde 1931, anciavam os professores das cadeiras de clinicas por instalações adequadas onde pudessem ministrar os ensinamentos de suas catedras com o conforto e com a eficiência necessárias ao bom aproveitamento dos alunos.

Os anos passavam, a população da Capital e do Estado a crescer vertiginosamente, o número de enfermos a aumentar paralelamente, as instalações emprestadas da Santa Casa de Misericórdia a se tornarem inadequadas e insuficientes.

Tal situação tornava-se insustentavel sob pena de S. Paulo perder seu título de Estado lider da União, pelo menos no tocante ao ensino médico e a assistência hospitalar.

Compreendeu-o muito bem o Dr. Adhemar de Barros, como médico e como filantropo, dando início a construção do Hospital das Clínicas, no dia 10 de Outubro de 1938. Obra gigantesca, de proporções desconhecidas na América do Sul, cobre uma área de terreno de 4.000 metros quadrados e a soma das áreas dos vários pavimentos perfaz o total de 42.000 metros quadrados de construção.

Possue 238 enfermarias, sendo 62 de 1 leito, 21 de 2,63 de 3 , 13 de 4,2 de 5,3 de 6,70 de 7,2 de 8,1 de 2 e 1 de 11 leitos, além de enfermarias de emergência que poderão elevar a capacidade total do hospital a 1.100 leitos. Existem 17 salas de operaçôes, sendo 8 de tipo regular e 9 grandes, e destas, 3 possuem arquibanquadas para espectadores e uma é do tipo Gudin para esterilisação integral.

Em cada enfermaria há um pequeno laboratório para exames rotineiros, sala de preleções, salas de professor e assistentes, copa e sala de serviço.

Há ainda secções especiais para microbologia e sorologia, histopatologia, química clínica, fisiopatologia, moléstia da nutrição, eletrocardiologia, radiologia e radioterapia, mecano e massoterapia helioterapia, endoscopia, especialmente esofago - gastro - bronco - pulmonar, esterilização geral, farmacia, gabinete odontológico, oficina ortopédica, central térmica para produção de vapor e de água quente, central elétrica, transformadores e gerador de emergência, depósitos e filtros dágua, desinfetório, frigorificos, lavanderia, cosinha geral e especial para dietas, rouparia e oficina de costura, oficina mecânica, . marcenaria, pintura, barbearià, vestiário para alunos, residência de médicos internos, de estagiarios e de empregados, fornos incineradores de lixo, etc., emfim, tudo quanto necessita um hospital moderno destinado não só ao tratamento dos doentes, como ao ensino médico e a pesquisa científica.

E' evidente que obra de tamanho vulto não pode completar-se em pequeno lapso de tempo sob pena de eivar-se de imperfeições.

Por outro lado, não sendo os recursos financeiros ilimitados é necessário que as verbas cubram vários orçamentos, acarretando dessarte algum retardamento na construção. 
Apezar desses impecilhos, é pensamento da diretoria da Faculdade inaugurar as secções de obstetricia, ginecologia, cirurgia geral. e ortopedica inclusive traumatologia e urologia, juntamente com as dependências administrativas necessárias ao funcionamento do hospital, em começo de Abril próximo futuro.

Já o Governo do ilustre Interventor Dr. Fernando Costa, destinou a quantia de 3.000 contos para terminar a construção e cogita de abrir o crédito necessário de pelo menos 10.000 contos para o equipamento.

Como complemento do hospital será fundada a Escola de Enfermagem de alto padrão, indispensavel para preparar enfermeiras a altura do serviço que lá deve ser prestado. Infelizmente, até este momento, a nobre profissão de enfermeira tem sido exercida entre nós por curiosas sem o devido preparo básico e sem a conveniente compreensão das altas finalidades das suas funções e do benefício que dai decorre para a sociedade.

Com a realisação deste conjunto, S. Paulo poderá ufanar-se de possuir o Centro Médico mais completo e mais moderno da América do Sul.

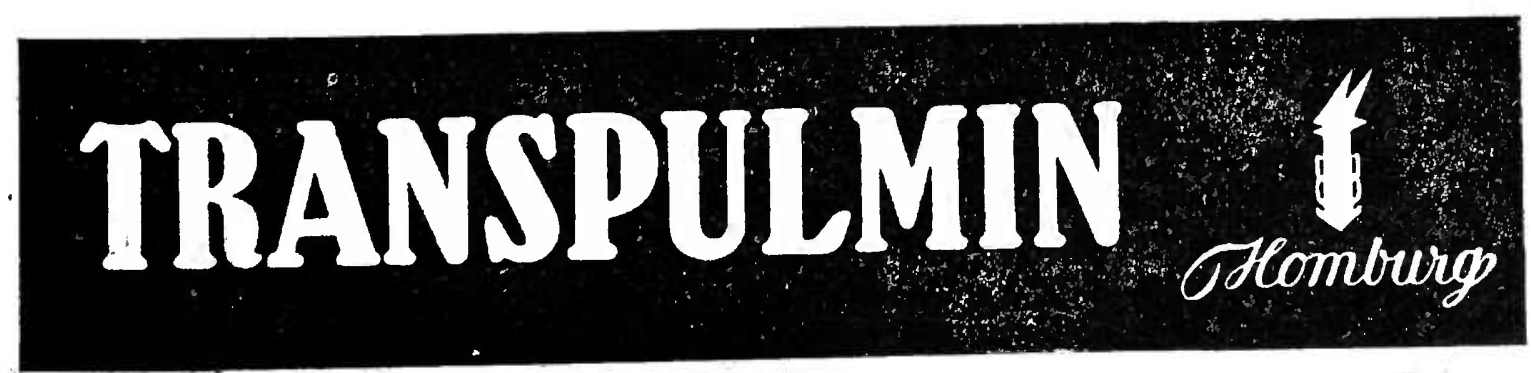




\section{Signalisação luminosa dos hospitaes COM O APARELHAMENTO}

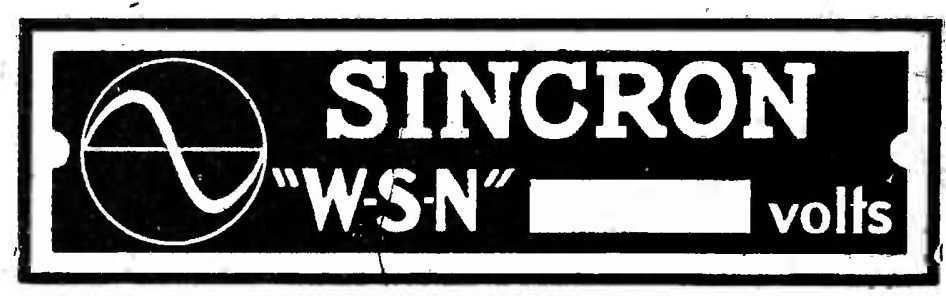

Os hospitais bem dirigidos do Brasil pensaram em seus doentes e em suas enfermeiras, dando-lhes o apparelhamento "Sincron" para chamadas de enfermeiras.

Entre estes, figuram os hospitaes:

Hospital das Clinicas da Faculdade de Medicina de São Paulo (São Paulo)

Maternidade Matarazzo - (São Paulo)

Sanatorio Jabaquara - (São Paulo)

Hospital Miguel Pereira, em Mandaqui - (São Paulo)

Santa Casa de Misericordia de São Paulo — varios pavilhões (São Paulo).

Casa de Saude Santa Rita - (São Paulo)

Hospital da Cruz Azul - (São Paulo)

Hospital Santa Catharina - 3. ${ }^{\circ}$ andar - (São Paulo)

Hospital de Santa Ignez - (São Paulo)

Hospital de Jaçanã - (São Paulo)

Maternidade de São Paulo - pavilhões novos - (São Paulo)

Maternidade de Jahú - (Jahú-S. Paulo)

Instituto Orthopedico Godoy Moreira - (São Paulo)

Circulo Operario Ypiranga - (São Paulo)

Hospital Esperança - parte nova - (São Paulo)

Hospital de Recife - (Pernambuco)

Hospital Irmãs Franciscanas - (Santa Cruz-R. G. Sul)

Hospital Central da Marinhá - (Ilha das Cobras)

Hospital Central do Exercito - (Rio de Janeiro)

Hospital Central de Accidentados - (Rio de Janeiro)

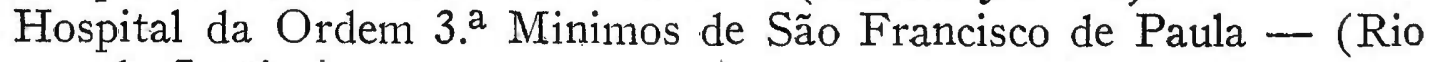
de Janeiro)

Instituto Nacional de Biologia - Pav. Marcilio Dias - (Rio de Janeiro)

Posto Medico Base Aviação Naval - (Ilha do Governador-Galeão)

Clinica do Dr. Moura Brasil - (Rio de Janeiro)

Clinica de Olhos Dr. Paulo Filho - (Rio de Janeiro)

Casa de Caridade Leopoldinense - (Leopoldina-Minas Geraes)

Hospital Funcionarios Publicos - (Rio de Janeiro)

Pedir catalogos illustrados e orçamentos de installação á

F A B R I C A S I N C R O N

Rua Cesario Ramalho, 158 - Tel. 7-3625 - SÃO PAULO 
TAMBEM NUM HOSPITAL É O REVESTIMENTO IMPERMEABILISADO - FACTOR MAIS IMPORTANTE, NÃO SÓ PARA A SAUDE DO PREDIO, QUANTO PARA O PROGRESSO DA CURA DOS INTERNADOS. PORTANTO EMPREGANDO-SE - IMPERMEABILLIS A N TE

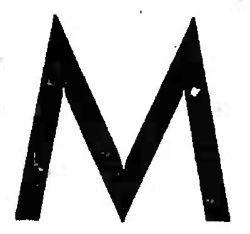

PARA A FACHADA E ALICERCES, ASSEGURA-SE ASSIM TODA A CONSTRUCÇÃO CONTRA A HUMIDADE POR TODOS OS TEMPOS. - DISTRIBUIDORES EM S. PAULO: CARVALHO MEIRA \& CIA. LTDA., V. ISNARD \& CIA LTDA., O. MATARAZZO \& CIA. LTDA., CIREX. LTDA. - EM BELLO HORIZONTE: C. I R. ROMEO DE PAOLI \& CIA. LTDA. - EM RIO DE JANEIRO: PARQUET PAULISTA LTDA :: :: :: : 


\section{Exc \\ .. exige alimento}

- estimulo à celula nervosa

\section{DE TRABALALHO}

Nergofon

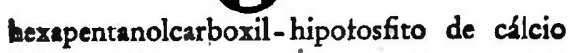

encerrando $35 \mathrm{mg}$. de fós-

foro elementar por ampôla

de 2 cmc., em combinação

organocálicica, exerce essa

dupla acão de maneira rápida e duradoura.

\section{AMOSTRAS}

disposição dos

Srs. Médicos
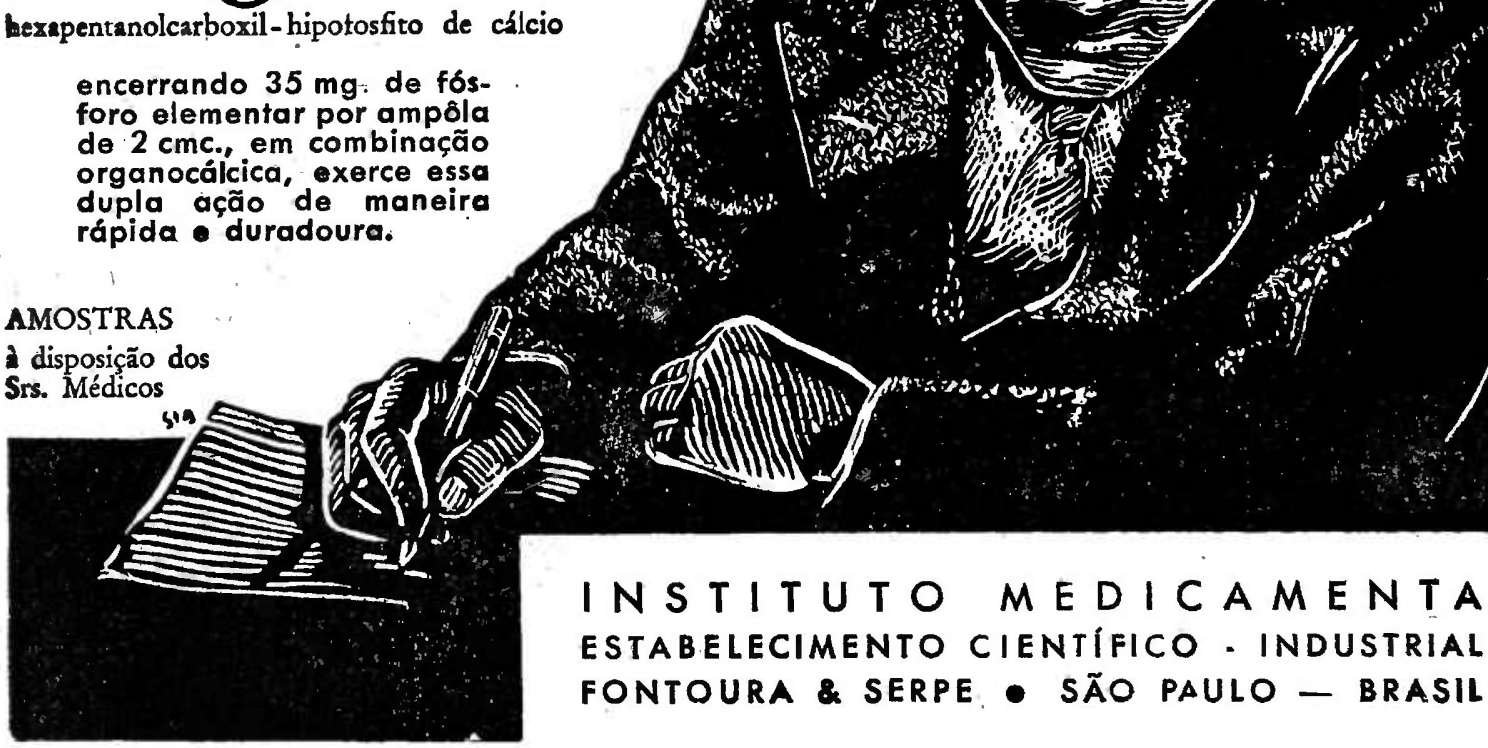

INSTITUTO MEDICA MENTA. ESTABELECIMENTO CIENTÍFICO - INDUSTRIAL FONTOURA \& SERPE • SÃO PAULO - BRASIL

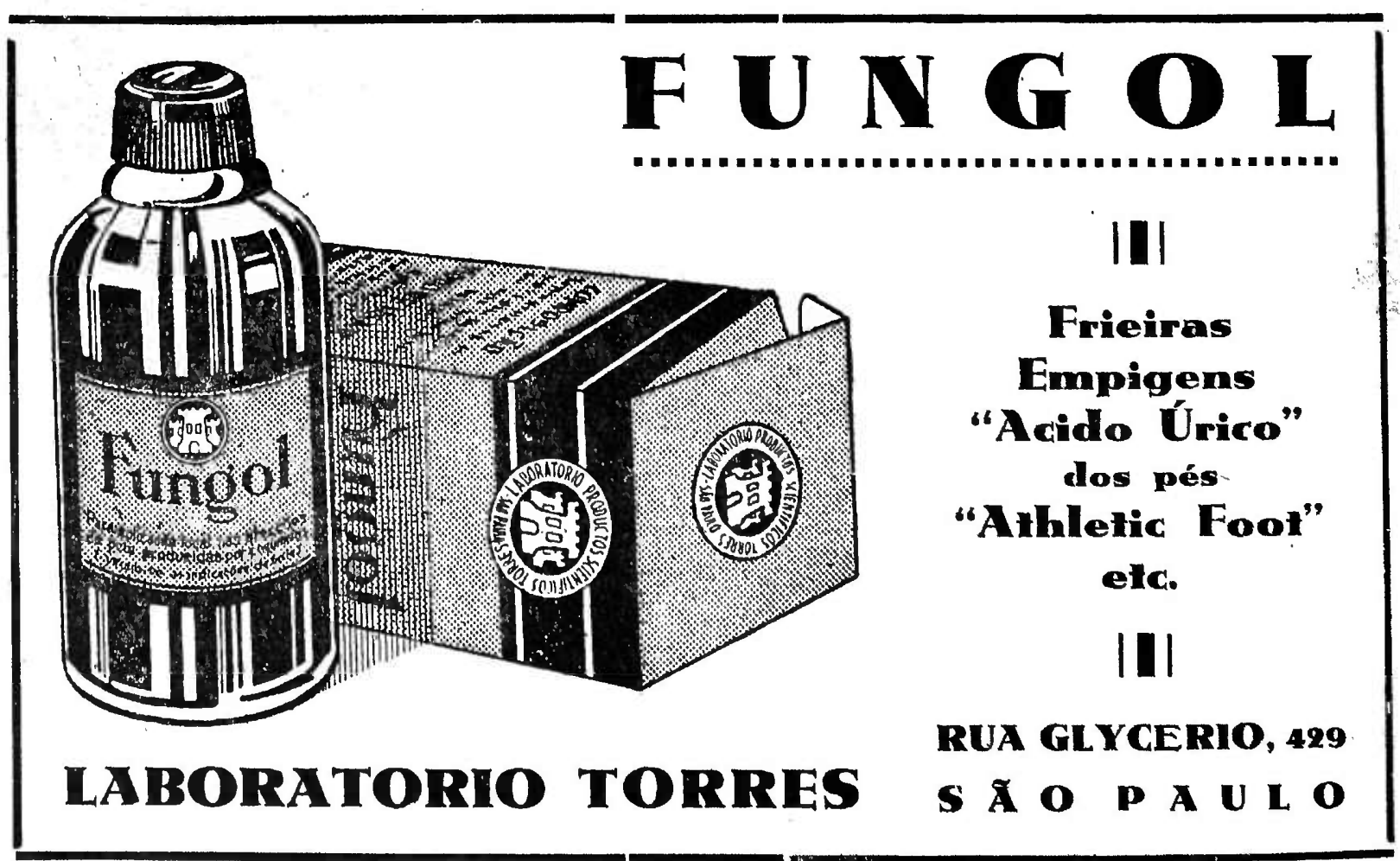




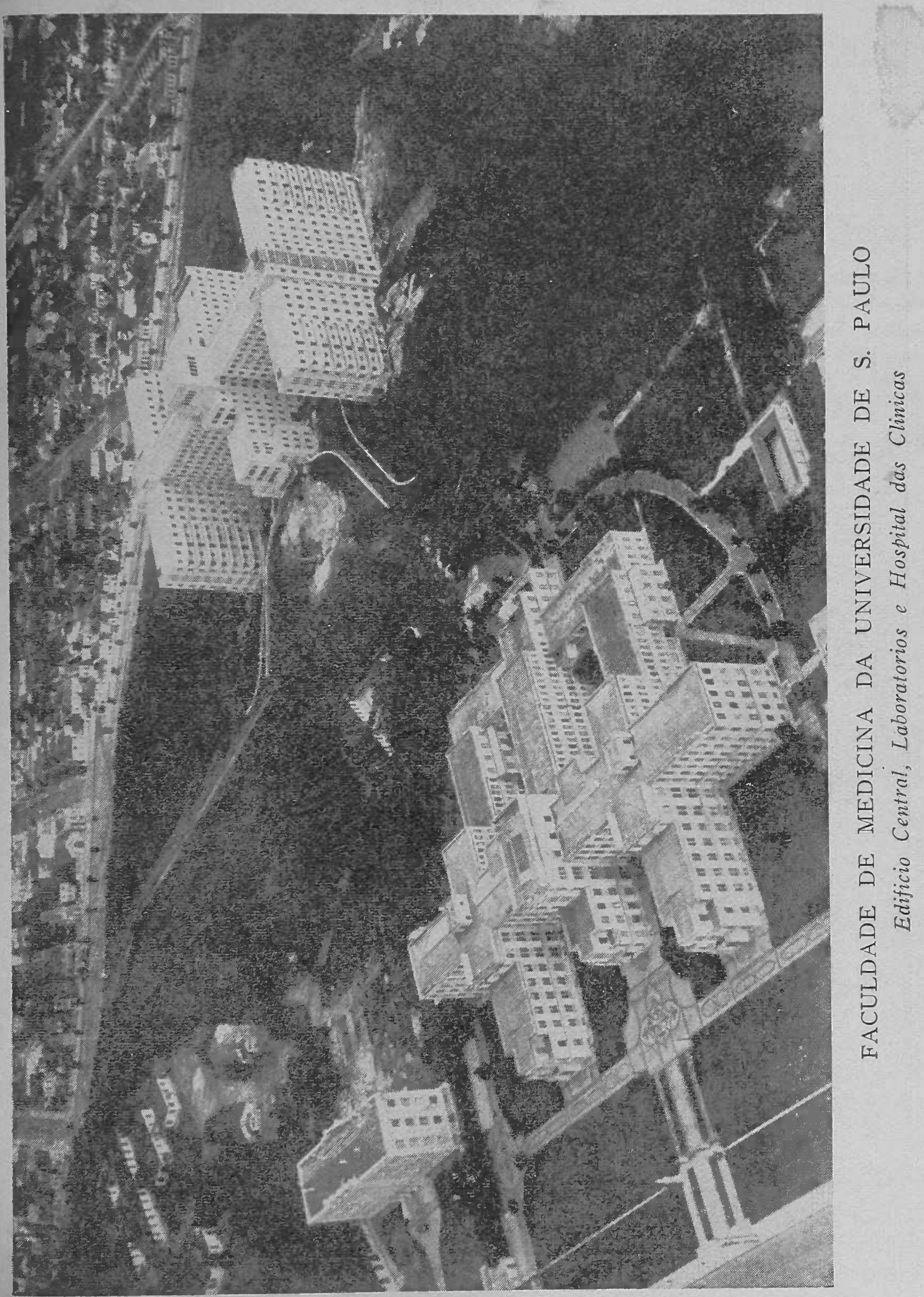




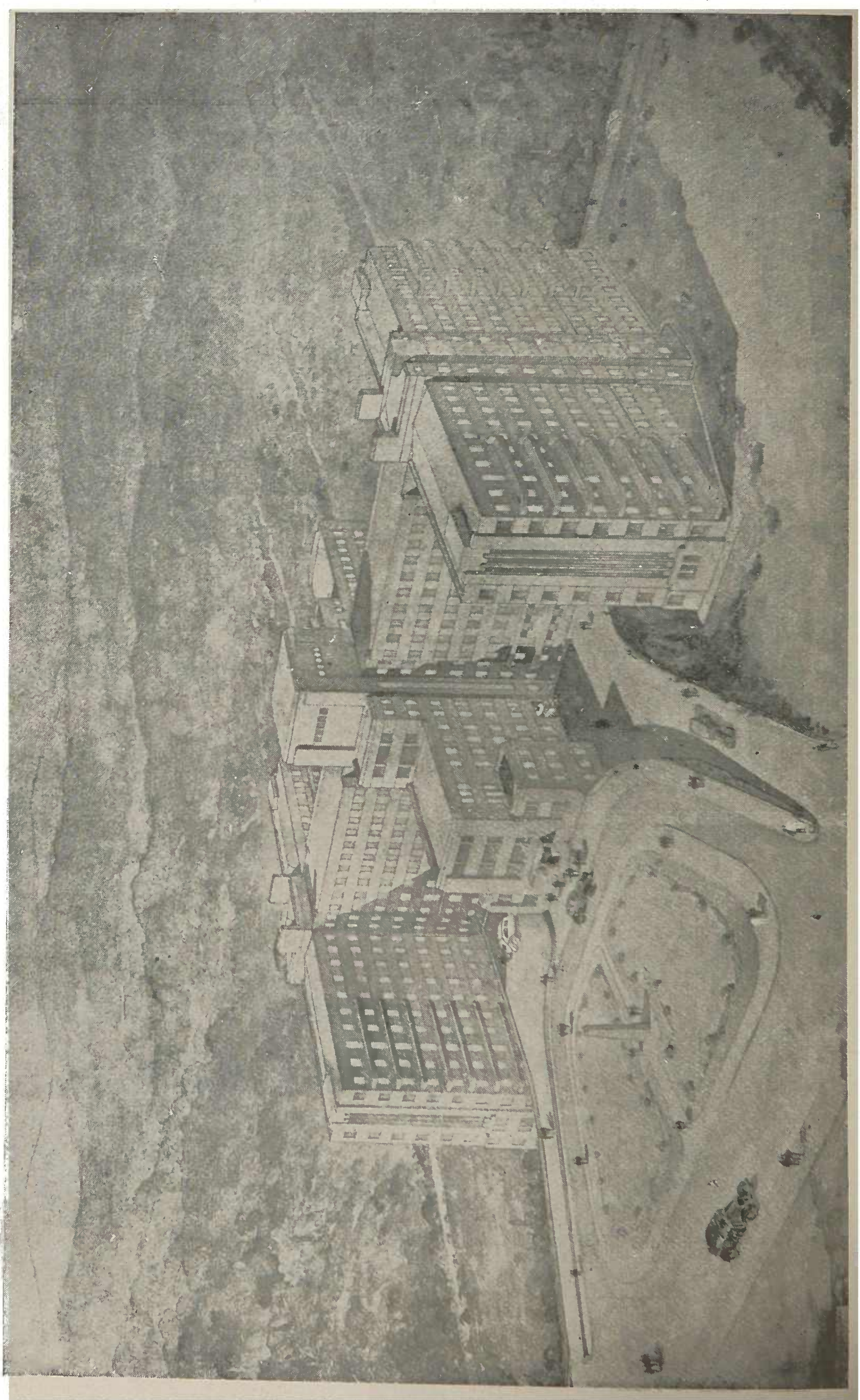




\section{Anticonvulsivo para o tratamento da epilepsia}

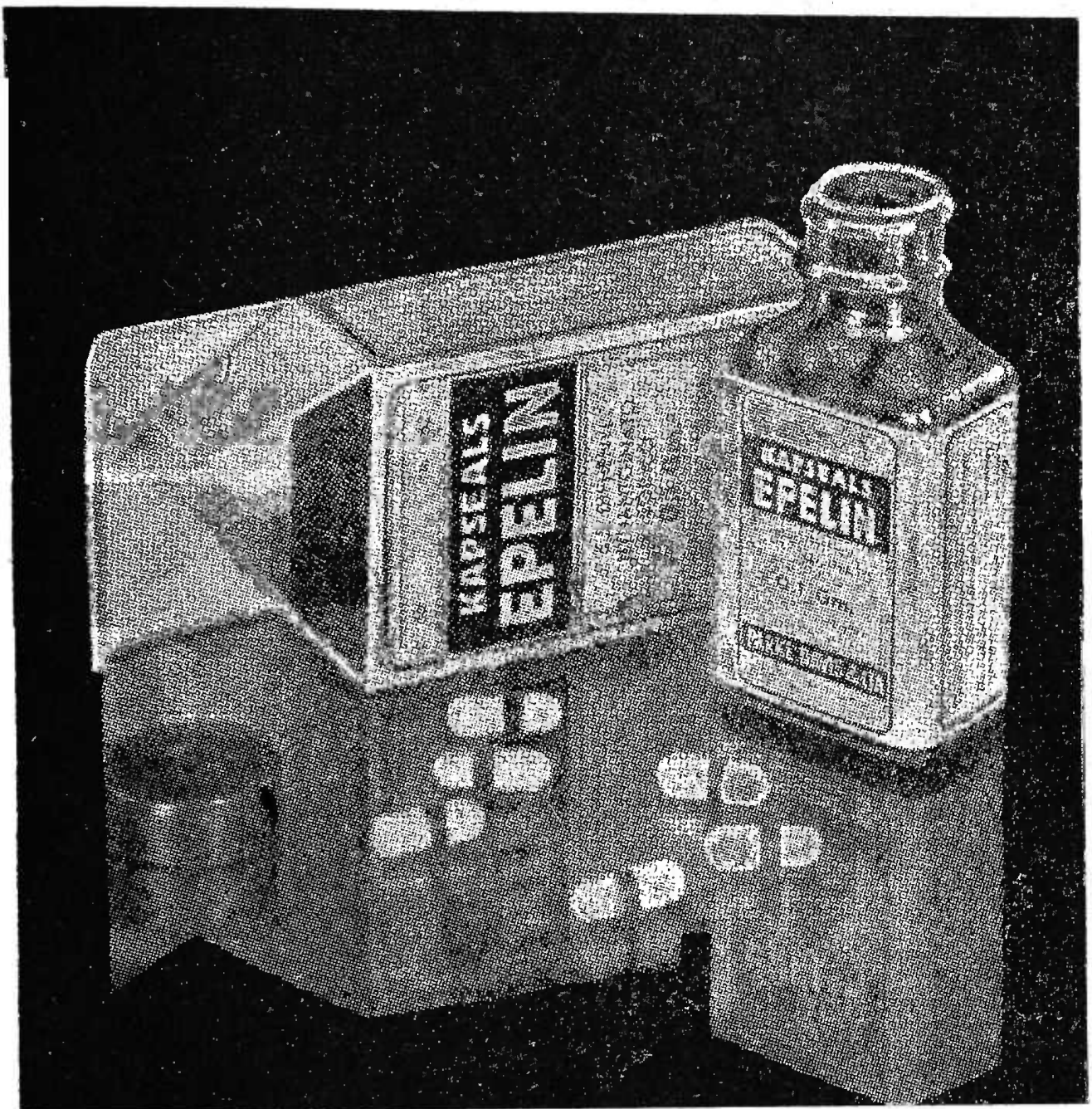

KAPSEALS

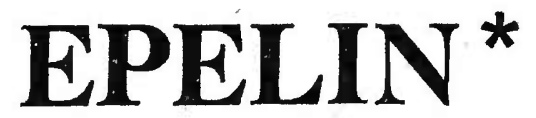

(5,5 - diphenyl hydantoinato sodico)

O PRODUTO ORIGINAL

E'PELIN é um novo anticonvulsivo, praticamente destituido de effeito hypnotico. Superior em actividade aos bromuretos e barbituricos, sem os inconvenientes destes. Previne ou diminue grandemente a incidencia e o rigor dos ataques convulsivos na maioria dos epilepticos.

EPELIN é indicado no tratamento das syndromes convulsivas e epilepsia, especialmente nos casos refractarios a outras medicações.

Apresentado em vidros de 30 Kapseạls (capsulas selladas) de 0,1 gm.

\section{Parke, Davis \& Companhia}

Rua Marquez de S. Vicente, 99/103

RIO DE JANEIRO

* EPELIN denomina-se DILANTIN SODIUM nos E. U. A. e EPANUTIN na Inglaterra, todos fabricados exclusivamente por Parke, Davis \& Cia. 
1

\section{Aumento acentuado}

e duradouro da pressão sanguínea

por elevação da quantidade de șangue circulante e do volume por minuto, sem aumento notavel da frequencia das contracções cardíacas e sem estrangulamento da irrigação sanguinea de orgãos de importancia vital, por meio do

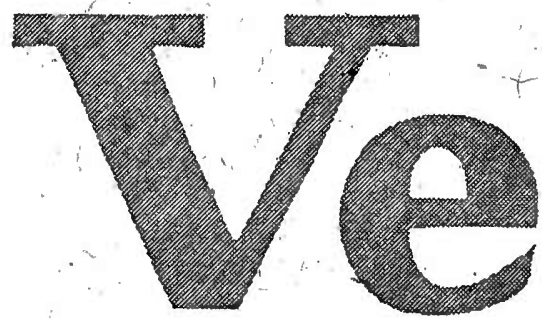

nOVO

\section{medicamento contra o colapso}

e estimulănte circulatorio

de acção periferica.

Estimula a circulação. fazendo reingressar nela o sangue venoso imobilizado em certos territorios O Veritol dilata os vasos coronarios e aumenta a eficiencia do trabalho cardiaco

\section{Efeito energico tambem por via oral.}

Líquido. Vidros de 10 c.c Empôlas. Caixas de 5 empôlas.

KNOLL A.-G., LUDWIGSHAFEN S/O RHENO (ALEMANHA). 